\title{
Therapeutic role of systematic lymphadenectomy in early-stage endometrial cancer: A systematic review
}

\author{
MEI-YI LI ${ }^{1,2}$, XIAO-XIA HU ${ }^{1}$, JIAN-HONG ZHONG ${ }^{3}$, LU-LU CHEN $^{1}$ and YONG-XIU LIN ${ }^{1}$ \\ ${ }^{1}$ Gynecology and Obstetrics Department, People's Hospital of Guangxi Zhuang Autonomous Region, \\ Guangxi Zhuang Autonomous Region 530021; ${ }^{2}$ Gynecology and Obstetrics Department,
}

Changsha Hospital for Maternal and Child Health Care, Changsha, Hunan 410007; ${ }^{3}$ Surgical Oncology Department, Affiliated Tumor Hospital of Guangxi Medical University, Nanning, Guangxi Zhuang Autonomous Region 530021, P.R. China

Received December 9, 2014; Accepted January 18, 2016

DOI: $10.3892 / 01.2016 .4467$

\begin{abstract}
The purpose of the current review was to examine whether systematic lymphadenectomy is safe and effective for treating early-stage endometrial cancer. PubMed, Embase, the Cochrane Library and the China National Knowledge Infrastructure databases were systematically searched during April 2014 to identify studies comparing the use of systematic lymphadenectomy and no systematic lymphadenectomy in parallel for the treatment of early-stage endometrial cancer. A total of 13 eligible studies involving 51,155 patients were included in this review. The median overall survival (OS) rate at 5 years following lymphadenectomy was $90 \%$ (range, 73.1-98.3\%) for patients undergoing the systematic procedure and $88.2 \%$ (range, 68-98.4\%) for patients not undergoing the systematic procedure. For the two types of lymphadenectomy, OS has tended to improve over the last 20 years. The combined rate of disease-free and progression-free survival was higher in patients who underwent systematic lymphadenectomy, and the recurrence rate was lower. In particular, systematic lymphadenectomy was associated with markedly higher OS than the non-systematic procedure for patients with intermediate- and high-risk endometrial cancer when $\geq 11$ lymph nodes were removed. Systematic lymphadenectomy demonstrates clinical benefit in patients with early-stage endometrial cancer and should thus be a standard treatment option. In conclusion,
\end{abstract}

Correspondence to: Professor Xiao-Xia Hu, Gynecology and Obstetrics Department, People's Hospital of Guangxi Zhuang Autonomous Region, 6 Tao Yuan Road, Nanning, Guangxi Zhuang Autonomous Region 530021, P.R. China

E-mail: huxxia@hotmail.com

Professor Jian-Hong Zhong, Surgical Oncology Department, Affiliated Tumor Hospital of Guangxi Medical University, $71 \mathrm{He}$ Di Road, Nanning, Guangxi Zhuang Autonomous Region 530021, P.R. China

E-mail: zhongjianhong66@163.com

Key words: lymphadenectomy, endometrial cancer, early-stage, overall survival systematic lymphadenectomy leads to higher OS than no systematic lymphadenectomy in intermediate- and high-risk patients with early-stage endometrial cancer, particularly when the procedure removes $\geq 11$ lymph nodes.

\section{Introduction}

Endometrial cancer is one of the most prevalent malignant tumors of the female genital tract, accounting for $6 \%$ of all cases of cancer in women (1). According to the American Cancer Society, the annual incidence of endometrial cancer is increasing, with $\sim 40,880$ new cases diagnosed in the USA in 2005 (2) and 49,560 new cases in 2013 (3). The median age of patients with endometrial cancer is 58 years, and the 5-year overall survival (OS) rate of those in the early stages of the disease is $80-85 \%$ (4). The high incidence and mortality rate highlight the necessity for optimized therapy for patients with endometrial cancer.

The guidelines of the International Federation of Gynecology and Obstetrics (FIGO), which were updated in 2009 to include a revised pathology surgical staging system (5), recommend surgical therapy involving total hysterectomy and bilateral salpingo-oophorectomy, peritoneal washing and pelvic and/or para-aortic lymphadenectomy. The guidelines of the National Comprehensive Cancer Network (NCCN) also recommend that surgical staging include pelvic and para-aortic lymphadenectomies for women with endometrial cancer (6). According to guidelines $(5,6)$, a lymphadenectomy should be performed when myometrial invasion is $>50 \%$, or in tumors of grade 3 or a special pathological type. Despite the FIGO and NCCN recommendations, the survival benefit of lymphadenectomy in early-stage endometrial cancer has not been clearly defined, and the extent and performance of lymphadenectomy varies from surgeon to surgeon. Several retrospective studies have suggested that a therapeutic benefit is associated with lymphadenectomy in early-stage endometrial cancer $(7,8)$; however, two large randomized control studies have failed to demonstrate a survival advantage $(9,10)$.

Similarly, the guidelines are lacking on how to treat pelvic and para-aortic lymph node metastases. Pelvic metastases may occur in up to $10 \%$ of patients with early-stage endometrial cancer, and para-aortic metastases may occur in as many as 
$6 \%$ (11). Although clinicians generally agree that surgery is the best curative treatment option, they may disagree regarding the optimal procedure to use.

Lymphadenectomy is widely used to assess whether endometrial cancer has metastasized and reduces the risk of lymphatic metastasis (8). Non-systematic lymphadenectomy usually involves removing < 11 lymph nodes (7). However, to try to treat and prevent metastases, physicians often perform systematic lymphadenectomy, which involves removing $\geq 11$ lymph nodes in the same area covered by non-systematic lymphadenectomy $(9,10)$. Systematic lymphadenectomy is significantly more invasive than the non-systematic procedure, and leaves patients with markedly lower postoperative lymph function, increasing the risk of various complications (12). Given the controversy regarding whether systematic lymphadenectomy is justified for patients with early-stage endometrial cancer, the present study aimed to examine its safety and efficacy by systematically reviewing the literature.

\section{Materials and methods}

Search strategy. The current systematic review was conducted in accordance with the recommendations of the PRISMA statement (13). PubMed (www.ncbi.nlm.nih.gov/pubmed), Embase (www.embase.com), the Cochrane Library (www.cochrane library.com), and the China National Knowledge Infrastructure (www.cnki.net) databases (accessed April 2014) were searched during April 2014, without language restrictions and using the following search terms: 'Lymphadenectomy', 'lymph nodes dissection', 'endometrial cancer', 'endometrial carcinoma', 'early stage' and 'low risk'.

Study selection and quality assessment. Randomized control trials (RCTs) and prospective or retrospective studies (non-RCTs) directly comparing systematic lymphadenectomy and no systematic lymphadenectomy in patients with clinically localized endometrial cancer were eligible for inclusion. Studies were included if they met the following criteria: i) They involved patients with pathologically proven early-stage endometrial cancer determined to be preoperative FIGO stage I or occult stage II disease; and ii) they provided data on Kaplan-Meier analysis of OS for patients who underwent surgical resection. Studies involving patients who were diagnosed with other malignant neoplasms were excluded.

The methodological quality of included RCTs was assessed according to the guidelines in the 2008 version of the Cochrane Handbook for Systematic Reviews of Interventions (14). RCTs were considered to be high-quality if they reported on three or four of the items, which included random allocation, concealment of random allocation, blinding of persons who assess treatment effects and intention-to-treat analysis. The methodological quality of the included non-RCTs was assessed using the star scoring system based on the Newcastle-Ottawa Scale (15), which examines the method used to select patients, the comparability of the study groups, and the number of outcomes reported. Non-RCTs were considered high-quality if they received $>7$ out of 10 possible stars.

Outcomes. The primary outcome of the current systematic review was OS. Data on recurrence-free survival (RFS), disease-free survival (DFS) and operative mortality and morbidity were captured as a secondary outcome where available. In addition, patients were classified into risk subgroups based on criteria from the Gynecologic Oncology Group and FIGO (5).

Data extraction. Data were extracted independently by two authors. Data included author details, publication year, preoperative staging, the number of patients, patient characteristics, interventions used and conclusions, using a data extraction form. Any discrepancies in data extraction were resolved by consensus.

Data synthesis. Owing to the clinical heterogeneity among studies, meta-analysis was not performed. Instead findings from each study were synthesized and evaluated qualitatively.

\section{Results}

Description of studies. A total of 2,184 potentially eligible studies were identified and, ultimately, 13 studies involving 51,155 patients were included in the review (Fig. 1). The total population included 20,676 patients in the systematic lymphadenectomy group and 30,479 patients in the no systematic lymphadenectomy group. Two of the 13 studies were RCTs $(9,10)$ while the remaining 11 were retrospective non-RCTs $(7,8,16-24)$. Study recruitment periods extended from 1969 to 2008, and the studies involved centers from Italy, the USA, Germany, South Korea, Canada and China. Two studies $(20,24)$, involving a total of 253 patients, did not report the numbers of lymph nodes dissected in the systematic lymphadenectomy and no systematic lymphadenectomy groups. All studies were in English, except for two that were in Chinese $(20,24)$. Two studies involved only the adenocarcinoma type of endometrial cancer $(22,23)$, whereas the other studies involved endometrial cancer of various pathological types.

A total of 1,487 patients from four studies $(10,17,22,24)$ underwent only pelvic lymph node dissection. As expected, the recurrence rate was higher in patients exhibiting greater numbers of cancer-positive lymph nodes or a greater extent of lymph node metastasis $(7-10,17,19,22,23)$. The median number of lymph nodes removed during systematic lymphadenectomy ranged from 11 to 30 . The key characteristics of the studies are presented in Table I.

Methodological quality. Quality assessment scores of the included studies are summarized in Tables II and III. The RCTs were considered to be high quality as they reported at least three items. Similarly, the non-RCTs were judged to be of moderate to high quality, scoring 7-9 stars.

Clinical characteristics. The clinical characteristics of the 13 studies comprising 51,155 patients are listed in Tables I and IV. In the absence of international standards regarding the difference between the two types of lymphadenectomy, systematic dissection was considered to involve the removal of $\geq 11$ lymph nodes (7-10,16-19,21-23), including the dissection of iliac, obturator $(17,18,20,22,24)$ and selective para-aortic lymph nodes $(7-10,16,19,21,23)$. The cases that were classed as not systematic lymphadenectomy consisted of 9 cases that did not undergo lymphadenectomy $(8,16-20,22-24), 3$ cases that 


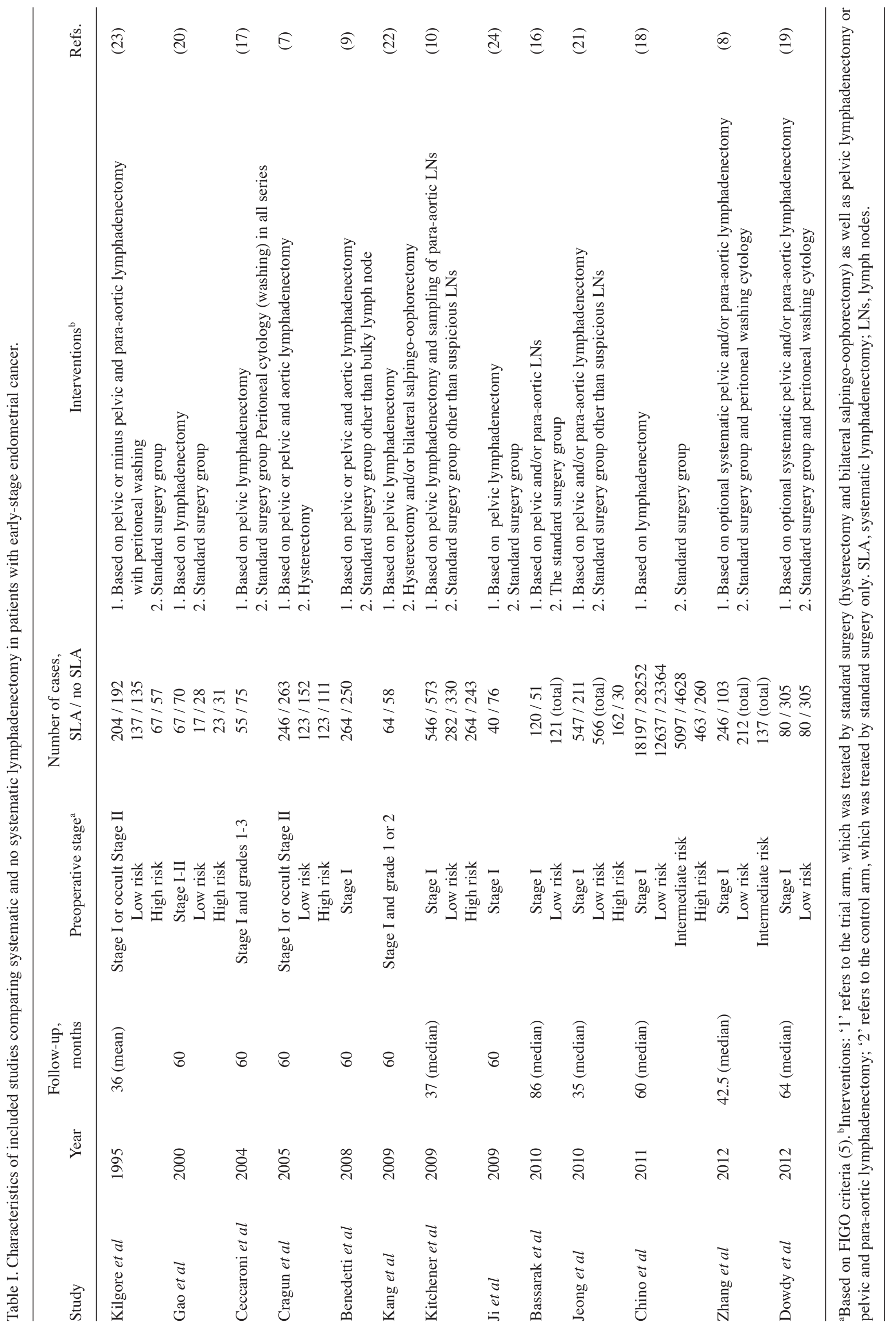


Table II. Assessment of methodological quality of randomized controlled trials included in the current review.

\begin{tabular}{|c|c|c|c|c|}
\hline Study & $\begin{array}{c}\text { Random allocation } \\
\text { (description of procedure) }\end{array}$ & $\begin{array}{l}\text { Concealment of } \\
\text { random allocation }\end{array}$ & $\begin{array}{l}\text { Blinding of persons who } \\
\text { assess treatment effects }\end{array}$ & $\begin{array}{l}\text { Intention-to-treat } \\
\text { analysis }\end{array}$ \\
\hline Benedetti et al (9) & + & + & + & + \\
\hline Kitchener et al (10) & + & + & + & - \\
\hline
\end{tabular}

Table III. Assessment of methodological quality of non-randomized trials included in the current review. ${ }^{\mathrm{a}}$

\begin{tabular}{|c|c|c|c|c|c|c|c|c|}
\hline \multirow[b]{2}{*}{ Study } & \multicolumn{3}{|c|}{ Patient selection $^{\mathrm{a}}$} & \multicolumn{2}{|c|}{ Group comparability $^{\mathrm{b}}$} & \multicolumn{2}{|c|}{ Outcome reporting ${ }^{\mathrm{c}}$} & \multirow[b]{2}{*}{ Stars $^{\mathrm{d}}$} \\
\hline & $\mathrm{S} 1$ & $\mathrm{~S} 2$ & $\mathrm{~S} 3$ & $\mathrm{C} 1$ & $\mathrm{C} 2$ & O1 & $\mathrm{O} 2$ & \\
\hline Kilgore et al (23) & $*$ & $*$ & $*$ & $*$ & $*$ & $*$ & $*$ & 7 \\
\hline Gao et al (20) & $*$ & $*$ & $*$ & $*$ & $*$ & $*$ & $*$ & 7 \\
\hline Ceccaroni et al (17) & $*$ & $*$ & $*$ & $*$ & $* *$ & $*$ & $* *$ & 9 \\
\hline Cragun et al $(7)$ & $*$ & $*$ & $*$ & $* *$ & $*$ & $*$ & $* *$ & 9 \\
\hline Kang et al (22) & $*$ & $*$ & $*$ & $*$ & $* *$ & $*$ & $*$ & 8 \\
\hline Ji et al (24) & $*$ & $*$ & $*$ & $*$ & $*$ & $*$ & $*$ & 7 \\
\hline Bassarak et al (16) & $*$ & $*$ & $*$ & $*$ & $*$ & $*$ & $*$ & 7 \\
\hline Jeong et al (21) & $*$ & $*$ & $*$ & $*$ & $* *$ & $*$ & $* *$ & 9 \\
\hline Chino et al (18) & $*$ & $*$ & $*$ & $*$ & $* *$ & $*$ & $*$ & 8 \\
\hline Zhang et al (8) & $*$ & $*$ & $*$ & $*$ & $* *$ & $*$ & $*$ & 7 \\
\hline Dowdy et al (19) & $*$ & $*$ & $*$ & $*$ & $* *$ & $*$ & $* *$ & 9 \\
\hline
\end{tabular}

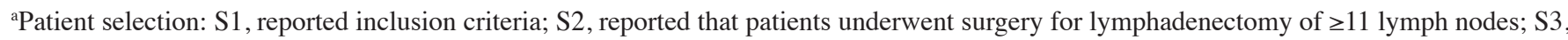
reported that patients underwent surgery without lymphadenectomy or with lymphadenectomy of $<11$ lymph nodes. ${ }^{\mathrm{b}} \mathrm{Group}$ comparability (if yes to all, 2 stars; if one of these characteristics was not reported, 1 star; if the two groups differed, no star): C1, age, body mass index and ethnicity; $\mathrm{C} 2$, tumor histological type, tumor grade, tumor stage, nodal dissection quantity. ${ }^{\mathrm{c}}$ Outcome reporting: O1, outcomes clearly reported (1 star); O2, quality of follow-up ( 2 stars if $>90 \%$ patients followed up for 5 years). ${ }^{\mathrm{d}}$ Out of a maximum possible 10 stars.

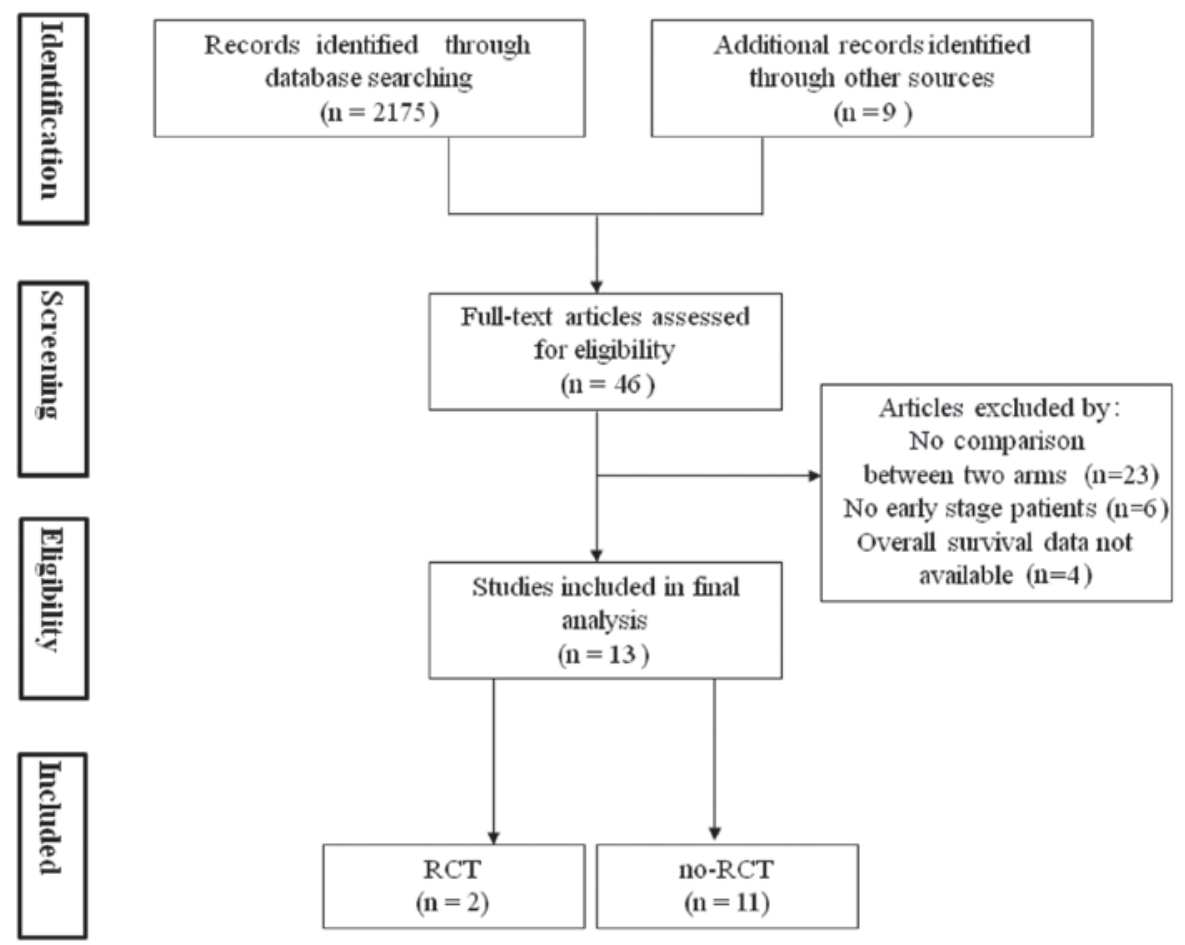

Figure 1. Selection of trials for a systematic review comparing systematic lymphadenectomy vs. no systematic lymphadenectomy in patients with early-stage endometrial cancer. 
involved the removal of suspicious lymph nodes $(9,10,21)$ and 1 case that involved the removal of $<11$ nodes (7). As the definitions of systematic and no systematic lymphadenectomy were different among all 13 studies, systematic vs. no systematic lymphadenectomy were defined using two criteria: Systematic dissection of lymphatic tissues vs. no lymphadenectomy other than suspicious lymph nodes or not performed; and removal of $<11$ vs. $\geq 11$ lymph nodes.

Association of disease-related survival associated with lymphadenectomy. Three studies $(7,16,22)$ reported data on progression-free survival (PFS), which was significantly higher for patients who underwent systematic lymphadenectomy of $\geq 11$ lymph nodes $(86 \%)$ compared with those who underwent no systematic lymphadenectomy $(75 \%$; $\mathrm{P}<0.001)(7)$. This difference in PFS was particularly notable among patients with intermediate- and high-risk disease. The other two retrospective studies reported similar PFS for the systematic lymphadenectomy and no systematic lymphadenectomy groups $(16,22)$.

One study reported that DFS was significantly higher for the group who underwent the systematic procedure involving $\geq 11$ lymph nodes $(88.1 \%)$ than for the no systematic procedure $(76.3 \% ; \mathrm{P}<0.05)$ patients with intermediate- and high-risk disease (8). The same study also found DFS to be similar for the systematic and no systematic procedures among patients with low-risk disease (91.2\% vs. $83.7 \%$; P>0.05). Another study identified DFS to be marginally higher following the systematic procedure for patients of mixed risk types, but this difference did not achieve statistical significance (9).

Two studies examined RFS. One study reported that systematic lymphadenectomy involving removal of $\geq 11$ lymph nodes was associated with lower RFS compared with no systematic lymphadenectomy, regardless of patient endometrial cancer risk classification (10). Another study (19) reported similar RFS for systematic lymphadenectomy and no systematic lymphadenectomy.

Taken together, these data suggest that systematic lymphadenectomy is associated with a higher combined rate of DFS and PFS and a lower recurrence rate when compared with no systematic lymphadenectomy (Tables IV and V).

Association of OS with lymphadenectomy. The 5-year OS rate, determined from 11 studies (7-9,15,16,18-23), ranged from 73.1 to $98.3 \%$ (median, $90 \%$ ) in the systematic lymphadenectomy group; this was higher than the rate in the no systematic lymphadenectomy group, which ranged from 68 to $98.4 \%$ (median, 88.2\%). In the majority of studies, however, the OS difference did not achieve statistical significance. Among non-RCTs, only six trials $(7,8,16,17,21,23)$ found the difference to be significant $(\mathrm{P}<0.05)$, and neither of the RCTs in the current review found the difference to be significant after analyzing subgroups with different risk classifications $(9,10)$. The number of mortalities ranged from 1 to 53 patients across all studies, while recurrence ranged from 2.4 to $13.0 \%$.

Subsequently, OS was investigated in subgroups of patients with different risk classifications. Seven studies reported subgroup data for a total of 38,489 patients with low-risk disease and 11,696 with intermediate- to high-risk disease $(7,8,10,18,20,21,23)$. The non-RCTs reported significantly higher OS rates for the systematic 
Table V. Summary of results comparing disease-related survival for SLA vs. no SLA.

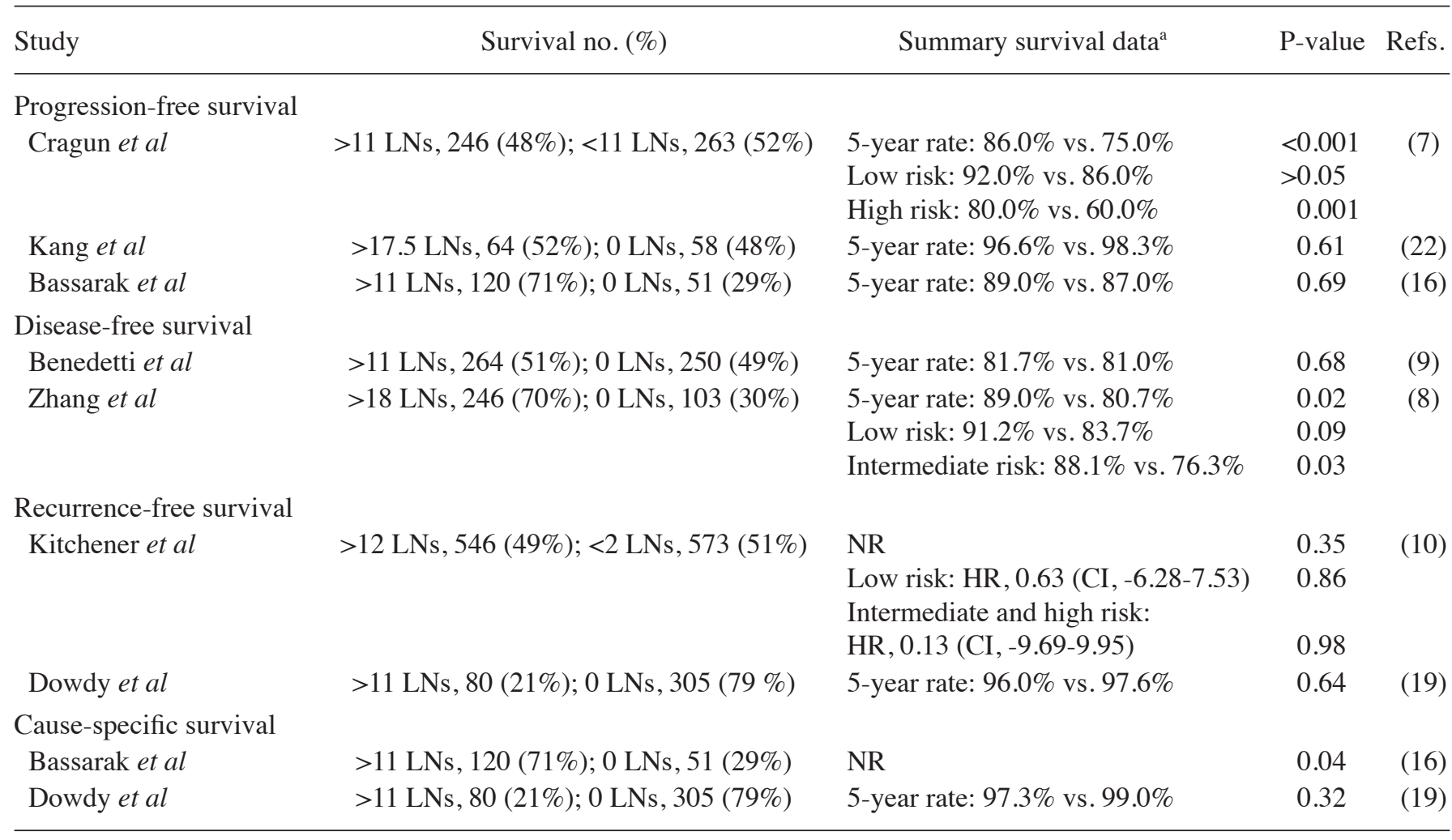

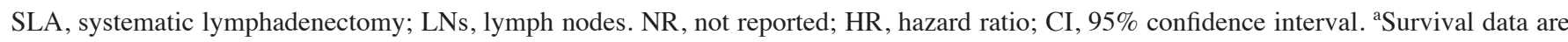
presented as systematic lymphadenectomy vs. no systematic lymphadenectomy and include all data.

lymphadenectomy than for the no systematic lymphadenectomy procedure in patients with intermediate- and high-risk disease $(\mathrm{P}<0.05)(7,8,18,21,23)$, while OS rates were similar for the two procedures among patients with low-risk disease. Data from these trials indicated a trend that removal of $\geq 11$ lymph nodes was associated with more favorable OS compared with removal of $<11$ lymph nodes $(7,8,18,21,23)$. The results for OS are summarized in Tables IV and VI.

Complications. The complications of lymphadenectomy were most often reported to be lymphocysts and lymphedema, both of which were reported in three studies $(8,9,22)$. In one of these studies, the two complications occurred in 35 of the 264 patients $(13.2 \%)$ in the systematic lymphadenectomy group, but in only 4 of the 250 patients (1.6\%) in the no systematic lymphadenectomy group (8). In the other two studies, however, the rates of these complications were similar between the two groups. Infrequent complications were directly related to surgery (e.g., wound infection and nerve or bladder injury) or the patient's medical condition (e.g., bowel obstruction, deep venous thrombus and pneumonia). No surgery-related mortalities were reported in any of the included studies. Furthermore, no long-term complications and none of the reported complications appeared to significantly affect long-term survival.

\section{Discussion}

Whether systematic or no systematic lymphadenectomy is more appropriate for patients in the early stages of endometrial cancer is controversial. Although the systematic procedure should, in principle, offer better protection against metastasis and recurrence, the current systematic review of studies involving a total of $>50,000$ patients from 6 countries suggests that systematic lymphadenectomy does not reliably improve survival time over no systematic lymphadenectomy when patients of all endometrial cancer risk classes are combined. In addition, the systematic procedure may be associated with higher rates of complications, including lymphedema and lymphocysts, which reduce postoperative quality of life and increase risk of mortality $(12,25)$.

In the enrolled studies, the results were not consistent. Certain studies reported no difference in OS rates between systematic and no systematic lymphadenectomy $(9,10)$, while other studies indicated that systematic lymphadenectomy improved OS $(7,8,16,17,21,23)$. These results must be interpreted carefully for a number of reasons. Firstly, it is possible that only a small proportion of patients in the systematic lymphadenectomy group underwent para-aortic lymphadenectomy; in one study, for example, the proportion was only $26 \%$ (9). Secondly, it is possible that a high proportion of patients in the systematic lymphadenectomy group did not, in fact, undergo such extensive dissection; in one study, for example, $60 \%$ of the systematic lymphadenectomy group underwent dissection of $<14$ lymph nodes, including the pelvic lymph nodes but not the para-aortic ones (10). Furthermore, systematic and no systematic lymphadenectomy were defined differently among the included studies; six studies presented a vague definition of systematic and no 


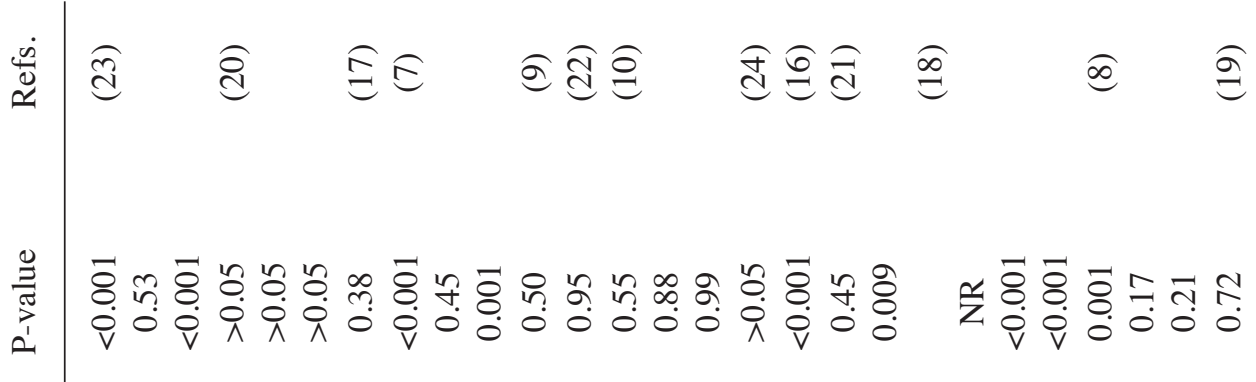

f.

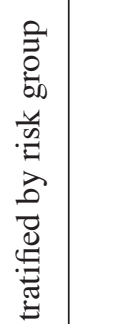

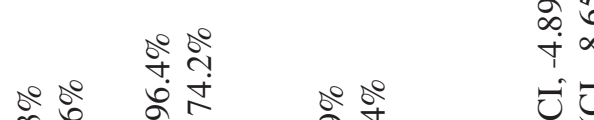

已ُ

它

के

象?

$\therefore$ 造

$\dot{\infty} \dot{\infty}$

0

并舀

नू०

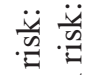

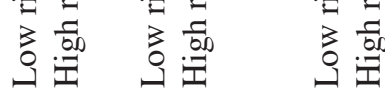

疍

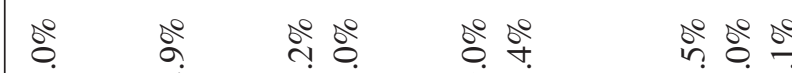

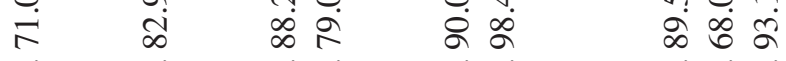

$\dot{s} \dot{p}$

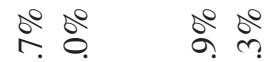

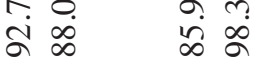

$\dot{\vec{p}} \dot{\vec{p}} \dot{\vec{p}}$

$\underset{\infty}{\infty}$

岂苛苛芯芯

离

离离品

응

के

这这这

离

离苛离

章

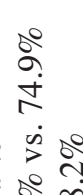

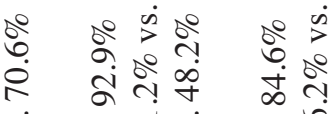

$\dot{s} \dot{s} \infty \dot{\infty} \dot{s} \dot{\phi} \dot{\infty}$

b. o

ब

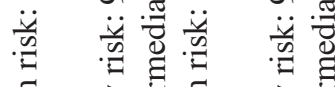

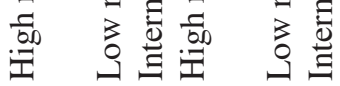

号号

艺市

เ

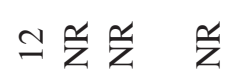

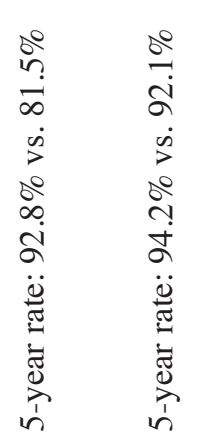

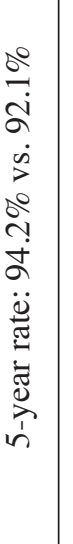

号一ナ

nิ $\sim \frac{x}{z}$

兑兑艺艺

q $=$

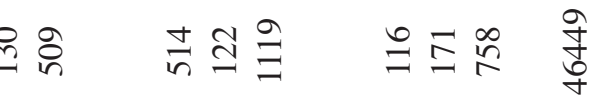

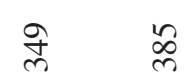

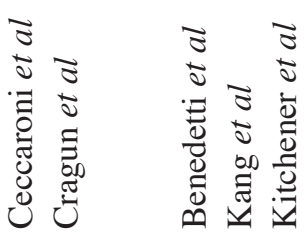

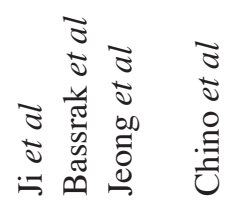

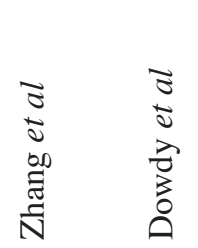


systematic lymphadenectomy $(9,10,16,18,20,24)$, while seven studies showed definite cut-off values of numbers of removed lymph nodes for defining the two types of lymphadenectomy $(7,8,17,19,21-23)$. As previous studies have emphasized that the number of removed lymph nodes affects survival in patients with endometrial cancer $(26,27)$, the diverse definitions of systematic lymphadenectomy may be the cause of the different efficacies of systematic lymphadenectomy for OS in patients with endometrial cancer.

The role of adjuvant radiation in early-stage endometrial cancer is also controversial. Eight of the enrolled studies, involving a total of 50,046 patients, reported the use of postoperative adjuvant radiotherapy $(7,9,10,16-18,21,23)$. Four studies $(7,16,18,23)$ observed that systematic lymphadenectomy in patients with grade III lesions and deep myometrial invasion and who were receiving postoperative adjuvant radiotherapy resulted in better survival than no systematic lymphadenectomy. However, four studies $(9,10,17,21)$ reported similar survival rates when comparing patients receiving adjuvant radiotherapy. There may be potential for bias due to adjuvant radiation blunting the effect of systematic lymphadenectomy. Therefore, future investigation is required to define the role of adjuvant radiation in early-stage endometrial cancer.

Nevertheless, the current analysis does provide evidence that systematic lymphadenectomy leads to higher OS rates than the no systematic procedure specifically in patients with intermediate- or high-risk early-stage endometrial cancer. Removing $\geq 11$ lymph nodes from these patients led to more favorable OS compared with the removal of a smaller number, regardless of the cancer status of the lymph nodes. However, no definitive guidelines are available regarding the number of lymph nodes that should be dissected. Although nodal count is indicative of the extent of lymphadenectomy, the number of nodes reported by the pathologist depends not only on anatomical variations in patients, but also on surgical expertise and the comprehensiveness of pathological analysis. Chan and Kapp (28) reported that systematic removal of all lymphatic tissue in the retroperitoneal region may be the most accurate definition of a complete lymphadenectomy. These findings suggest that systematic lymphadenectomy to remove $\geq 11$ lymph nodes, or even a complete systematic removal of all lymphatic tissue, may be appropriate therapy for patients with intermediate- or high-risk early-stage endometrial cancer.

Metastasis of endometrial cancer to the lymph nodes worsens prognosis and increases the risk of lymphatic recurrence (29); $90 \%$ of patients who suffer such recurrence succumb to the disease (30). One study reported 5-year survival rate of $90 \%$ for patients with stage I endometrial cancer, compared to only $54 \%$ for patients with metastatic stage III disease (31).

Several studies suggest that lymph node resection is more reliable than surgery alone to determine whether endometrial cancer has metastasized and to reduce the risk of metastasis $(10,11,32)$. Although one study reported that only $4.6 \%$ of patients with low-risk disease (stage I, grade 1) have lymph node metastases (32), other studies have reported that $98 \%$ of patients with para-aortic involvement have grossly positive pelvic nodes (11), and that $9 \%$ of stage I patients have positive pelvic lymph nodes while $6 \%$ have positive para-aortic nodes (33). Although several histopathological risk factors have been demonstrated to correlate closely with lymph node metastasis, including tumor grade, depth of myometrial invasion and the presence of $30-50 \%$ serous or clear cell histology (34), lymphadenectomy remains the most direct way to assess and reduce risk of metastasis. Previous studies reported that high-risk patients who undergo para-aortic lymphadenectomy as part of their surgical staging procedure exhibit higher survival rates than those who undergo simple surgical staging $(35,36)$.

The current systematic review suggests that systematic lymphadenectomy should be applied carefully to patients with early-stage endometrial cancer, as it is unlikely to produce clinical benefits in a high proportion of patients. A similar story emerges from systematic reviews of lymphadenectomy to treat for other malignancies, including prostatic cancer $(37)$ and gastric cancer $(38,39)$. These reviews come to divergent conclusions, and the overall evidence of clinical benefit is weak. This highlights the need for more prospective, high-quality, controlled studies of the safety and efficacy of systematic lymphadenectomy, in the context not only of endometrial cancer but of other cancers as well.

While this systematic review contributes significantly to the literature by examining a large number of patients in several countries and ethnic groups, its findings are nevertheless subject to several limitations. Firstly, only two of the included trials are RCTs, while the remaining 11 are retrospective non-RCTs. Secondly, patient selection, techniques used to dissect lymph nodes and perform surgery, and postoperative care and follow-up varied substantially across the included studies, which weakens the strength of the conclusions. Thirdly, a previous meta-analysis (40) has shown that, in one of the included trials (26), which accounts for 12,333 of the patients in the population included the present study, body mass index varies significantly between the systematic and no systematic lymphadenectomy groups and that controlling for this leads to a significantly higher OS rate for the systematic lymphadenectomy group than for the no systematic lymphadenectomy group. This highlights the requirement for future studies to control for all possible confounders when examining the clinical effects of systematic lymphadenectomy.

In conclusion, the available evidence suggests that systematic lymphadenectomy may improve OS in patients with intermediate- or high-risk early-stage endometrial cancer, particularly when $\geq 11$ lymph nodes are removed; however, this may not be the case in patients with low-risk disease. In addition, systematic lymphadenectomy may be associated with higher rates of lymphocysts and lymphedema. These findings argue that the use of systematic lymphadenectomy in carefully selected patients may improve endometrial cancer staging, choice of adjuvant therapy and prognosis prediction.

\section{Acknowledgements}

This work was supported by the National Natural Science Foundation of China (project no. 81060219).

\section{References}

1. Bray F, Loos AH, Oostindier M and Weiderpass E: Geographic and temporal variations in cancer of the corpus uteri: Incidence and mortality in pre- and postmenopausal women in Europe. Int J Cancer 117: 123-131, 2005. 
2. Jemal A, Murray T, Ward E, Samuels A, Tiwari RC, Ghafoor A Feuer EJ and Thun MJ: Cancer statistics, 2005. CA Cancer J Clin 55: 10-30, 2005.

3. Siegel R, Naishadham D and Jemal A: Cancer statistics, 2013. CA Cancer J Clin 63: 11-30, 2013.

4. Sant M, Aareleid T, Berrino F, Bielska Lasota M, Carli PM, Faivre J, Grosclaude P, Hédelin G, Matsuda T, Møller H, et al: EUROCARE-3: Survival of cancer patients diagnosed 1990-94-results and commentary. Ann Oncol 14 (Suppl 5): S61-S118, 2003.

5. Creasman W: Revised FIGO staging for carcinoma of the endometrium. Int J Gynaecol Obstet 105: 109, 2009.

6. Greer BE, Koh WJ, Abu-Rustum N, Bookman MA, Bristow RE, Campos S, Cho KR, Copeland L, Eifel P, Jaggernauth W, et al: Uterine cancers. J Natl Compr Canc Netw 4: 438-462, 2006.

7. Cragun JM, Havrilesky LJ, Calingaert B, Synan I, Secord AA, Soper JT, Clarke-Pearson DL and Berchuck A: Retrospective analysis of selective lymphadenectomy in apparent early-stage endometrial cancer. J Clin Oncol 23: 3668-3675, 2005.

8. Zhang H, Jia L, Zhang Q, Zhang Y, Yang X and Kong B: Therapeutic role of systematic retroperitoneal lymphadenectomy in endometrial cancer. Bull Cancer 99: E10-E17, 2012.

9. Benedetti Panici P, Basile S, Maneschi F, Alberto Lissoni A, Signorelli M, Scambia G, Angioli R, Tateo S, Mangili G Katsaros D, et al: Systematic pelvic lymphadenectomy vs. no lymphadenectomy in early-stage endometrial carcinoma: Randomized clinical trial.J Natl Cancer Inst 100: 1707-1716, 2008.

10. ASTEC study group, Kitchener H, Swart AM, Qian Q, Amos C and Parmar MK: Efficacy of systematic pelvic lymphadenectomy in endometrial cancer (MRC ASTEC trial): A randomised study. Lancet 373: 125-136, 2009.

11. Morrow CP, Bundy BN, Kurman RJ, Creasman WT, Heller P, Homesley HD and Graham JE: Relationship between surgical-pathological risk factors and outcome in clinical stage I and II carcinoma of the endometrium: A Gynecologic Oncology Group study. Gynecol Oncol 40: 55-65, 1991.

12. Achouri A, Huchon C, Bats AS, Bensaid C, Nos C and Lécuru F: Complications of lymphadenectomy for gynecologic cancer. Eur J Surg Oncol 39: 81-86, 2013.

13. Moher D, Liberati A, Tetzlaff J and Altman DG; PRISMA Group: Preferred reporting items for systematic reviews and meta-analyses: The PRISMA statement. Ann Intern Med 151: 264-269, 2009.

14. Green S, Higgins JPT, Alderson P, Clarke M, Mulrow CD and Oxman AD: Cochrane Handbook for Systematic Reviews of Interventions. Version 5.0.2. The Cochrane Book Series, John Wiley \& Sons, Ltd., Hoboken, NJ, 2008.

15. Stang A: Critical evaluation of the Newcastle-Ottawa scale for the assessment of the quality of nonrandomized studies in meta-analyses. Eur J Epidemiol 25: 603-605, 2010.

16. Bassarak N, Blankenstein T, Brüning A, Dian D, Bergauer F, Friese $\mathrm{K}$ and Mylonas I: Is lymphadenectomy a prognostic marker in endometrioid adenocarcinoma of the human endometrium? BMC Cancer 10: 224, 2010.

17. Ceccaroni M, Savelli L, Bovicelli A, Dian D, Bergauer F, Friese K and Mylonas I: Prognostic value of pelvic lymphadenectomy in surgical treatment of apparent stage I endometrial cancer. Anticancer Res 24: 2073-2078, 2004.

18. Chino JP, Jones E, Berchuck A, Secord AA and Havrilesky LJ: The influence of radiation modality and lymph node dissection on survival in early-stage endometrial cancer. Int J Radiat Oncol Biol Phys 82: 1872-1879, 2012.

19. Dowdy SC, Borah BJ, Bakkum-Gamez JN, Weaver AL, McGree ME, Haas LR, Keeney GL, Mariani A and Podratz KC: Prospective assessment of survival, morbidity and cost associated with lymphadenectomy in low-risk endometrial cancer. Gynecol Oncol 127: 5-10, 2012.

20. Gao Y, Yu A and Chen L: Place of value of pelvic lymphadenectomy for treatment of endometrial neoplasms. Zhonghua $\mathrm{Fu}$ Chan Ke Za Zhi 35: 264-266, 2000 (In Chinese).

21. Jeong NH, Lee JM, Lee JK, Kim MK, Kim YJ, Cho CH, Kim SM, Park SY, Park CY and Kim KT: Role of systematic lymphadenectomy and adjuvant radiation in early-stage endometrioid uterine cancer. Ann Surg Oncol 17: 2951-2957, 2010.
22. Kang WD, Kim CH, Cho MK, Kim JW, Kim YH, Choi HS and Kim SM: Lymphadenectomy for low-risk endometrial cancer based on preoperative and intraoperative assessments. Int J Gynecol Cancer 19: 657-661, 2009.

23. Kilgore LC, Partridge EE, Alvarez RD, Austin JM, Shingleton HM, Noojin F III and Conner W: Adenocarcinoma of the endometrium: Survival comparisons of patients with and without pelvic node sampling. Gynecol Oncol 56: 29-33, 1995.

24. Ji RY, Gao N, Li Y, Yang Y, Liu S and Yao X: Clinical analysis of 116 cases of early stage endometrial cancer. Chinese Journal of Clinical Oncology 36: 917-919, 2009.

25. Angioli R, Plotti F, Cafà EV, Dugo N, Capriglione S, Terranova C, Montera R, Guzzo F and Panici PB: Quality of life in patients with endometrial cancer treated with or without systematic lymphadenectomy. Eur J Obstet Gynecol Reprod Biol 170: 539-543, 2013.

26. Chan JK, Cheung MK, Huh WK, Osann K, Husain A, Teng NN and Kapp DS: Therapeutic role of lymph node resection in endometrioid corpus cancer: A study of 12,333 patients. Cancer 107: 1823-1830, 2006

27. Lutman CV, Havrilesky LJ, Cragun JM, Secord AA, Calingaert B, Berchuck A, Clarke-Pearson DL and Soper JT: Pelvic lymph node count is an important prognostic variable for FIGO stage I and II endometrial carcinoma with high-risk histology. Gynecol Oncol 102: 92-97, 2006.

28. Chan JK and Kapp DS: Role of complete lymphadenectomy in endometrioid uterine cancer. Lancet Oncol 8: 831-841, 2007.

29. Mariani A, Webb MJ, Keeney GL, Aletti G and Podratz KC: Predictors of lymphatic failure in endometrial cancer. Gynecol Oncol 84: 437-442, 2002.

30. Mariani A, Webb MJ, Keeney GL and Podratz KC: Routes of lymphatic spread: A study of 112 consecutive patients with endometrial cancer. Gynecol Oncol 81: 100-104, 2001.

31. Lurain JR, Rice BL, Rademaker AW, Poggensee LE, Schink JC and Miller DS: Prognostic factors associated with recurrence in clinical stage I adenocarcinoma of the endometrium. Obstet Gynecol 78: 63-69, 1991.

32. Abu-Rustum NR, Iasonos A, Zhou Q, Oke E, Soslow RA, Alektiar KM, Chi DS and Barakat RR: Is there a therapeutic impact to regional lymphadenectomy in the surgical treatment of endometrial carcinoma? Am J Obstet Gynecol 198: 457, e451-455, 2008 (Discussion 457, e455-456).

33. Creasman WT, Morrow CP, Bundy BN, Homesley HD, Graham JE and Heller PB: Surgical pathologic spread patterns of endometrial cancer. A Gynecologic Oncology Group Study. Cancer 60 (8 Suppl): S2035-S2041, 1987.

34. Creasman WT, Mutch DE and Herzog TJ: ASTEC lymphadenectomy and radiation therapy studies: Are conclusions valid? Gynecol Oncol 116: 293-294, 2010.

35. Mariani A, Webb MJ, Galli L and Podratz KC: Potential therapeutic role of para-aortic lymphadenectomy in node-positive endometrial cancer. Gynecol Oncol 76: 348-356, 2000.

36. Todo $\mathrm{Y}$, Kato $\mathrm{H}$, Kaneuchi $\mathrm{M}$, Watari $\mathrm{H}$, Takeda $\mathrm{M}$ and Sakuragi N: Survival effect of para-aortic lymphadenectomy in endometrial cancer (SEPAL study): A retrospective cohort analysis. Lancet 375: 1165-1172, 2010.

37. Gao L, Yang L, Lv X, Bu S, Wan F, Qian S, Wei Q, Han P and Fan T: A systematic review and meta-analysis of comparative studies on the efficacy of extended pelvic lymph node dissection in patients with clinically localized prostatic carcinoma. J Cancer Res Clin Oncol 140: 243-256, 2014.

38. Wang Z, Chen JQ and Cao YF: Systematic review of D2 lymphadenectomy versus D2 with para-aortic nodal dissection for advanced gastric cancer. World J Gastroenterol 16: $1138-1149,2010$

39. Brar SS, Seevaratnam R, Cardoso R, Law C, Helyer L and Coburn N: A systematic review of spleen and pancreas preservation in extended lymphadenectomy for gastric cancer. Gastric Cancer 15 (Suppl 1): S89-S99, 2012.

40. Kim HS, Suh DH, Kim MK, Chung HH, Park NH and Song YS: Systematic lymphadenectomy for survival in patients with endometrial cancer: A meta-analysis. Jpn J Clin Oncol 42: 405-412, 2012 